\title{
Self-Consistent Diffraction Stress Analysis Method for Estimating Stress, Strain-Free Lattice Parameter and Composition of Solid Solutions
}

\author{
Takashi Harumoto*, Ji Shi and Yoshio Nakamura \\ Department of Materials Science and Engineering, Tokyo Institute of Technology, Tokyo 152-8552, Japan
}

Self-consistent diffraction stress analysis method is proposed for analyzing solid solutions. Owing to the feedback of the strain-free lattice parameter, it is possible to perform the proposed method even when the exact composition is unknown. Employing an example specimen of (111) fiber-textured palladium cobalt alloy film with different compositions, the validity of the proposed method is confirmed by comparing results with that of the conventional method. This convenient proposal expands the applicability of diffraction stress analysis. [doi:10.2320/matertrans.MT-M2020163]

(Received May 21, 2020; Accepted July 29, 2020; Published September 25, 2020)

Keywords: diffraction stress analysis, strain-free lattice parameter, composition estimation, textured film

\section{Introduction}

The importance of diffraction stress analysis is rapidly increasing, as it can be performed non-destructively and provides a reliable stress value(s) even if the specimen is under operation, nano-sized, or has a complex structure, such as layered. ${ }^{1-8)}$

For performing diffraction stress analysis, elastic constants that convert strain into stress are required, since the diffraction measurement only provides strain. Such constants are known as "diffraction" elastic constants and are indeed key parameters for analysis. In the case of pure and famous materials, diffraction elastic constants can be found in the literature or may be estimated from single-crystal elastic constants reported in the handbook on elasticity. ${ }^{9)}$ In contrast, for the case of most of alloys and solid solution ceramics, such literatures are absent or very limited and incomplete. Accordingly, the elastic constants may be estimated using the elasticity theory or ab-initio calculations considering its composition. Therefore, the exact composition is required, i.e. the composition measurement should be performed prior to diffraction stress analysis. In our view this requirement significantly narrows the applicability and convenience of diffraction stress analysis.

In light of this situation, we propose a self-consistent diffraction stress analysis method for analyzing the stress, strain-free lattice parameter and composition of solid solutions. This method is based on feedback of the strainfree lattice parameter into input. This feedback enables one to perform diffraction stress analysis on solid solutions of which exact compositions are unknown. The validity of the proposed method is experimentally confirmed using a rather difficult example of (111) fiber-textured palladium cobalt (PdCo) alloy films, an attractive material due to its interesting magnetic property changes under hydrogen loading, and therefore can be applied to hydrogen sensors. ${ }^{10-17)}$

This paper consists of five sections; $\S 1$ introduction (this section); $\$ 2$ brief review of the conventional method; $\$ 3$ proposal of the self-consistent method, $\S 4$ application example and $\S 5$ conclusion.

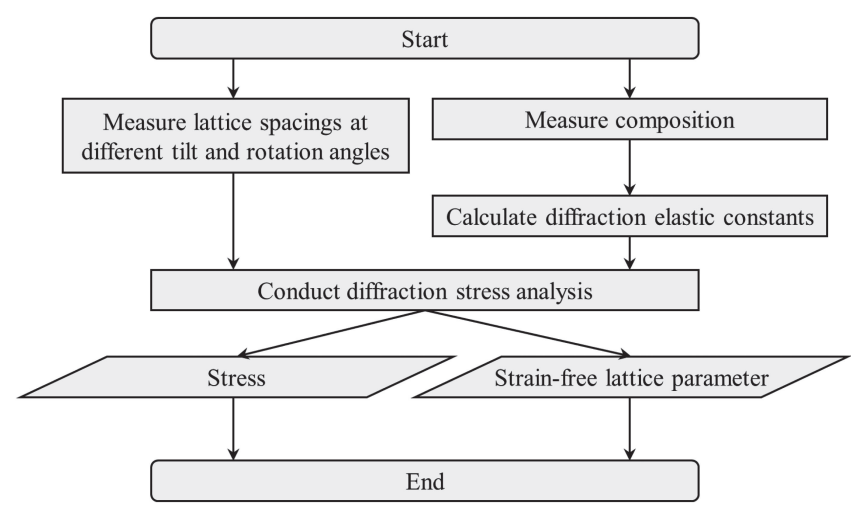

Fig. 1 Flowchart of the conventional diffraction stress analysis method.

\section{Conventional Diffraction Stress Analysis Method}

Prior to introducing the self-consistent method, the conventional diffraction stress analysis method is briefly reviewed in this section. Figure 1 explains the analysis flow.

The input data consists of two parts; a set of the lattice spacings measured at different tilt and rotation angles, and diffraction elastic constants. The diffraction elastic constants can be estimated experimentally from measuring lattice spacing changes due to the (known) applied stress. The alternative way, which may be more popular, is calculating diffraction elastic constants from single-crystal elastic constants by employing the appropriate grain interaction model. In the case of alloys, because single-crystal elastic constants of alloys are very limited (or not available), they may be estimated from single-crystal elastic constants of pure metals by considering its composition. The same situation can be found in solid solution ceramics. Therefore, the exact composition should be known before performing diffraction stress analysis.

After diffraction stress analysis, values of the stress and strain-free lattice parameter are determined. In general, great attention has been paid to the stress while perhaps surprisingly very little to the strain-free lattice parameter. 


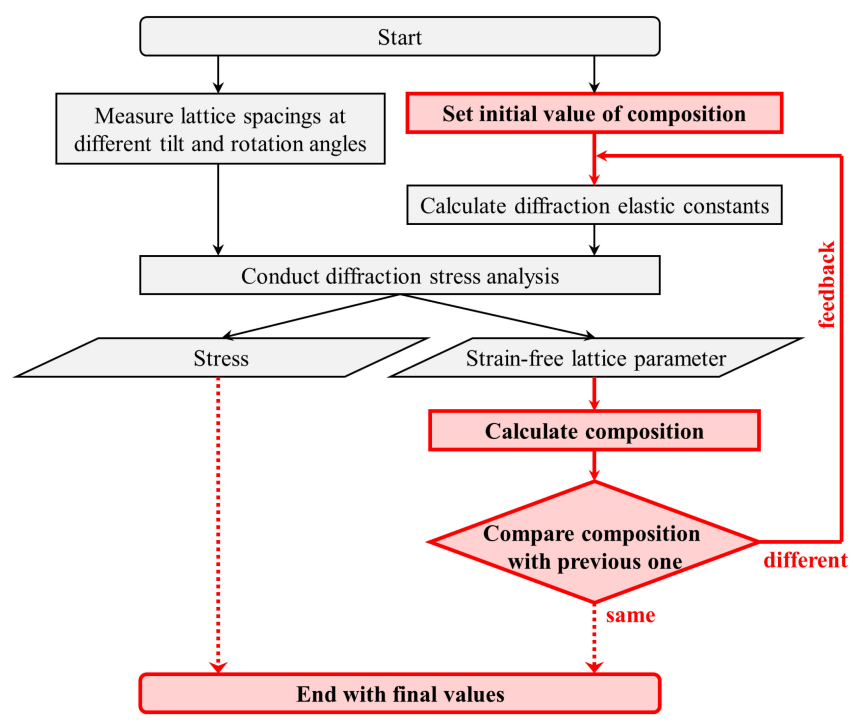

Fig. 2 Flowchart of the self-consistent diffraction stress analysis method. Differences are highlighted in red thick lines.

\section{Self-Consistent Diffraction Stress Analysis Method}

\subsection{Concept and analysis process}

The main concept of the proposed method is feedback of the strain-free lattice parameter. In this procedure, diffraction stress analysis can be performed even if the exact composition is unknown.

Details of the analysis flow is shown in Fig. 2. At the first (initial) cycle, since the exact composition is unknown, a chosen some number is inputted as the initial composition. From the initial composition, diffraction elastic constants are calculated and the diffraction stress analysis is performed. Next, the obtained result of the strain-free lattice parameter is fed back to the input in the form of the composition. From this composition the second cycle of the diffraction stress analysis is performed in the same manner. Self-consistency can be achieved after a sufficient cycle of feedbacks. In the end, values of the stress, strain-free lattice parameter and composition are obtained simultaneously. Note that the reliability of the strain-free lattice parameter is high as shown later (\$4.2.3).

\subsection{Applicability and requirements}

This self-consistent diffraction stress analysis method can be performed on solid solutions such as alloys and solid solution ceramics. The minimum requirements for conducting this analysis are the following;

(i) the strain-free lattice parameter can be obtained from diffraction stress analysis,

(ii) the strain-free lattice parameter can be converted into composition,

(iii) finally, from the composition, diffraction elastic constants can be estimated.

In general, requirement (i) can be attained easily. This is because, using the estimated stress value, it is possible to calculate the stress-free lattice spacing which can then be converted into the strain-free lattice parameter. In the case of a simple $\sin ^{2} \psi$ case (i.e. no texture), the strain-free lattice parameter can be easily estimated by considering the strain-free direction $\left(\sin ^{2} \psi^{*}\right.$, a direction which has no stress dependence, see Fig. S1 in supplementary figures in appendix). Regarding requirement (ii), the relation between the composition and strain-free lattice parameter can be found through the phase diagram. If it is unavailable, it is possible to acquire the lattice constant in a crystallographic database, such as ICDD PDF, and estimate the relation using it. Another choice is a first-order approximation, namely Vegard's law, although its reliability should be carefully considered. Requirement (iii) would be a little difficult, since the composition dependence of elastic constants is available only on famous materials. Accordingly, elastic constants may be estimated using the elasticity theory based on Morse potential $^{18)}$ or ab-initio calculation, ${ }^{19,20)}$ and converted into diffraction elastic constants. Therefore, the applicability of the proposed self-consistent diffraction stress analysis method is very wide, and most solid solutions can be analyzed. This method may be powerful for films because of the difficulty in the composition measurement on films in some cases.

\section{Application Example with (111) Fiber-Textured PdCo Alloy Films}

\subsection{Experimental}

\subsubsection{Specimen preparation}

(111) fiber-textured fcc PdCo solid solution alloy films with six different compositions (hereafter denoted as specimens A, B, C, D, E and F) were prepared using magnetron sputter deposition. The details of the deposition apparatus are in our previous report. ${ }^{21)}$ The films were deposited on polished synthetic fused silica glass (thickness: $0.5 \mathrm{~mm}$ ) at room temperature. The layer structure of films is a single layer of $100-\mathrm{nm}$-thick PdCo, i.e. $\mathrm{PdCo}(100 \mathrm{~nm}) /$ glass substrate, and no upper/under layer was introduced. Note that the film thickness of $100 \mathrm{~nm}$ is sufficiently thick to exclude the thin film effect which may have an influence on the lattice constant. Also, in this study, the substrate deformation may be relatively small and can be ignored, since the thickness of the substrate $(0.5 \mathrm{~mm})$ is much larger than the film thickness $(100 \mathrm{~nm})$. After the deposition, annealing at $673 \mathrm{~K}$ in vacuum was conducted to remove possible incorporated gas atoms, such as sputtering argon (Ar) gas, which may increase the lattice parameter. The exact composition of the prepared films was measured using Auger electron spectroscopy (AES). The peak intensities of AES peaks were corrected using relative sensitivity factors (RSFs).

\subsubsection{X-ray diffractometry}

X-ray diffraction measurement was conducted using a diffractometer equipped with a rotating copper $(\mathrm{Cu})$ anode, mirrors for beam parallelization, a pin-hole collimator $(0.1 \mathrm{~mm})$, a goniometer and a two-dimensional position sensitive detector (a distance from the goniometer center: $200 \mathrm{~mm}$ ). The divergence of the parallelized $\mathrm{Cu} K \alpha$ X-ray beam is smaller than $0.04^{\circ}$ in any direction, and the accuracy of the diffraction angle $2 \theta$ is better than $0.04^{\circ} .111$ and $11 \overline{1}$ peaks at $\psi=0^{\circ}$ and $70.5^{\circ}$, respectively, were measured. Peaks were fitted by two (superimposed) Lorentzian peaks representing $K \alpha_{1}$ and $K \alpha_{2}$. The error in the obtained peak 
center position was assumed to be a summation of following two components: one is $0.04^{\circ}$ originated from the diffractometer $2 \theta$ angle accuracy and another is the fitting error in the peak center position calculated during the peak fitting process. In this study, the former error was relatively large compared to the latter. All parameters calculated from the peak center position were shown with errors estimated from the error in the peak center position by considering error propagation. In-plane rotational symmetry and fibertexture were confirmed by the pole figures. The in-plane symmetry of the stress in film was checked by measuring the in-plane rotation angle $\left(\phi: 0^{\circ}-360^{\circ}\right)$ dependence of $11 \overline{1}$ peak positions.

\subsubsection{Self-consistent diffraction stress analysis}

The strain $\left(\varepsilon_{\psi}{ }^{111}\right)$ equation for (111) textured film with cubic crystal structure under rotational symmetric biaxial stresses $\left(\sigma_{11}=\sigma_{22}=\sigma_{\|}\right.$and $\sigma_{33}=0$ where $\sigma_{11}$ and $\sigma_{22}$ are stresses along film in-plane directions and $\sigma_{33}$ is a stress along normal direction to film) is expressed simply as ${ }^{1,2,22-26)}$

$$
\varepsilon_{\psi}^{111}=\frac{d_{\psi}^{111}-a_{\mathrm{sf}} / \sqrt{3}}{a_{\mathrm{sf}} / \sqrt{3}}=\left(2 S_{1}^{111}+\frac{1}{2} S_{2}^{111} \sin ^{2} \psi\right) \sigma_{\|},
$$

where $d_{\psi}^{111}$ is the (111) lattice spacing at a tilt angle $\psi$, $a_{\mathrm{sf}}$ is the strain-free lattice parameter, and $S_{1}^{111}$ and $\frac{1}{2} S_{2}^{111}$ are diffraction elastic constants for 111 . Using single-crystal elastic compliances $\left(s_{11}, s_{12}\right.$ and $\left.s_{44}\right)$, two elastic constants are described as $(1 / 3) s_{11}+(2 / 3) s_{12}-(1 / 6) s_{44}$ and $(1 / 2) s_{44}$.

Since composition dependence on elastic compliances (or stiffnesses) of PdCo alloy could not be found in literature and ab-initio calculation cannot reproduce the elastic constant of fcc-Co, ${ }^{27)}$ we employed the simple assumption that the elastic properties of $\mathrm{Pd}_{100-x} \mathrm{Co}_{x}$ alloy can be approximated from the weight composition (mass\%) weighted average,

$$
c_{\mathrm{ij}}(x \text { in mass } \% \mathrm{Co})=\frac{(100-x)}{100} c_{\mathrm{ij}}^{\mathrm{Pd}}+\frac{x}{100} c_{\mathrm{ij}}^{\mathrm{Co}},
$$

where $c_{\mathrm{ij}}{ }^{\mathrm{Pd}}$ and $c_{\mathrm{ij}}^{\mathrm{Co}}$ are single-crystal elastic stiffnesses of face-centered cubic (fcc)-Pd and fcc-Co, respectively. This assumption is based on the report ${ }^{28}$ ) that Young's modulus of $\mathrm{PdCo}$ alloy is almost linear to mass\% (the deviation is only $2 \%$ or less) even though the magnetic transition takes place at low Co composition. Note that this linear behavior in mass \% turns into the quadratic relationship in the atomic composition (at \%), which is typical tendency reported in ab-initio calculations. ${ }^{19,20)}$ From the literature, ${ }^{27,29)} c_{11}{ }^{\mathrm{Pd}}$, $c_{12}{ }^{\mathrm{Pd}}, c_{44}{ }^{\mathrm{Pd}}, c_{11}{ }^{\mathrm{Co}}, c_{12}{ }^{\mathrm{Co}}$ and $c_{44}{ }^{\mathrm{Co}}$ are $227,176,72,225,160$ and $92 \mathrm{GPa}$, respectively. The estimated $c_{\mathrm{ij}}$ are converted into $s_{\mathrm{ij}}$ using the following relations ${ }^{1)}$

$$
\begin{aligned}
& s_{11}=\frac{\left(c_{11}+c_{12}\right)}{\left(c_{11}-c_{12}\right)\left(c_{11}+2 c_{12}\right)}, \\
& s_{12}=\frac{-c_{12}}{\left(c_{11}-c_{12}\right)\left(c_{11}+2 c_{12}\right)}, \\
& s_{44}=\frac{1}{c_{44}} .
\end{aligned}
$$

According to our previous investigation, ${ }^{30,31)}$ eq. (1) can be analytically solved using two measured (111) lattice spacings at $\psi=0^{\circ}$ and $70.5^{\circ}\left(d_{\psi=0^{\circ}}^{111}\right.$ and $\left.d_{\psi=70.5^{\circ}}^{11 \overline{1}}\right)$ as

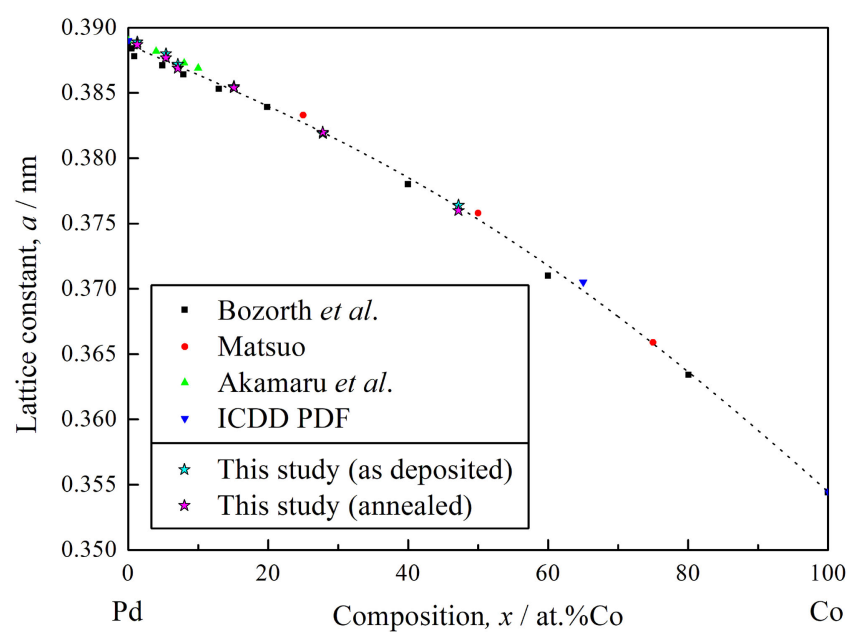

Fig. 3 Lattice constant of fcc- $\mathrm{Pd}_{100-x} \mathrm{Co}_{x}$ solid solution alloy in literatures. The fitted result described as eq. (8) is shown as a dashed line. The strainfree lattice constant estimated from the conventional method is also shown (however, it is not employed for estimating eq. (8)).

$$
\begin{aligned}
& \sigma_{\|}=-\frac{9}{2} \frac{d_{\psi=0^{\circ}}^{111}-d_{\psi=70.5^{\circ}}^{11 \overline{1}}}{\left(9 S_{1}^{111}+4 \frac{1}{2} S_{2}^{111}\right) d_{\psi=0^{\circ}}^{111}-9 S_{1}^{111} d_{\psi=70.5^{\circ}}^{11 \overline{1}}}, \\
& a_{\mathrm{sf}}=\sqrt{3}\left[\left(\frac{9}{4} \frac{S_{1}^{111}}{\frac{1}{2} S_{2}^{111}}+1\right) d_{\psi=0^{\circ}}^{111}-\frac{9}{4} \frac{S_{1}^{111}}{\frac{1}{2} S_{2}^{111}} d_{\psi=70.5^{\circ}}^{11 \overline{1}}\right] .
\end{aligned}
$$

Composition estimation from the strain-free lattice parameter was performed using the following relation

$$
\begin{aligned}
a(x \text { in at } \% \mathrm{Co})= & 1.0922 \times 10^{-10} x^{4}-2.3870 \times 10^{-8} x^{3} \\
& +1.7595 \times 10^{-7} x^{2}-2.3037 \times 10^{-4} x \\
& +0.38870 .
\end{aligned}
$$

This relation is estimated from fitting to literature values of bulk $\mathrm{Pd}_{100-x} \mathrm{Co}_{x}$ alloy ${ }^{10,32-35)}$ and shown as a dashed line in Fig. 3. When the composition difference between the present and previous is smaller than 0.1 at $\%$, we considered self-consistency to be achieved. The program code for this analysis is written in Microsoft Excel Visual Basic application (vba) and available on request. Note that a simple description is preferred and the code is not optimized for fast calculation (although it will finish within a second).

\subsection{Results and Discussion}

\subsubsection{Film structure and composition}

Figure 4 shows XRD profiles measured along $\psi=0^{\circ}$, i.e. normal $\theta-2 \theta$ scan. It is clear that only strong fcc 111 and 222 peaks are detected while other peaks are absent (upon close examination, however, a small trace of a 200 peak can be found in specimen A). Thus, the prepared films have (111) texture, the most typical texture for fcc metal film deposited onto glass substrate. This texture is enhanced by annealing at $673 \mathrm{~K}$. The 111 texture is further confirmed from pole figures (Fig. S2). The strong intensity spot at the center $\left(\psi=0^{\circ}\right)$ and the ring intensity distribution at around $\psi=70^{\circ}$ demonstrate the fiber-texture. The rotational symmetry of 


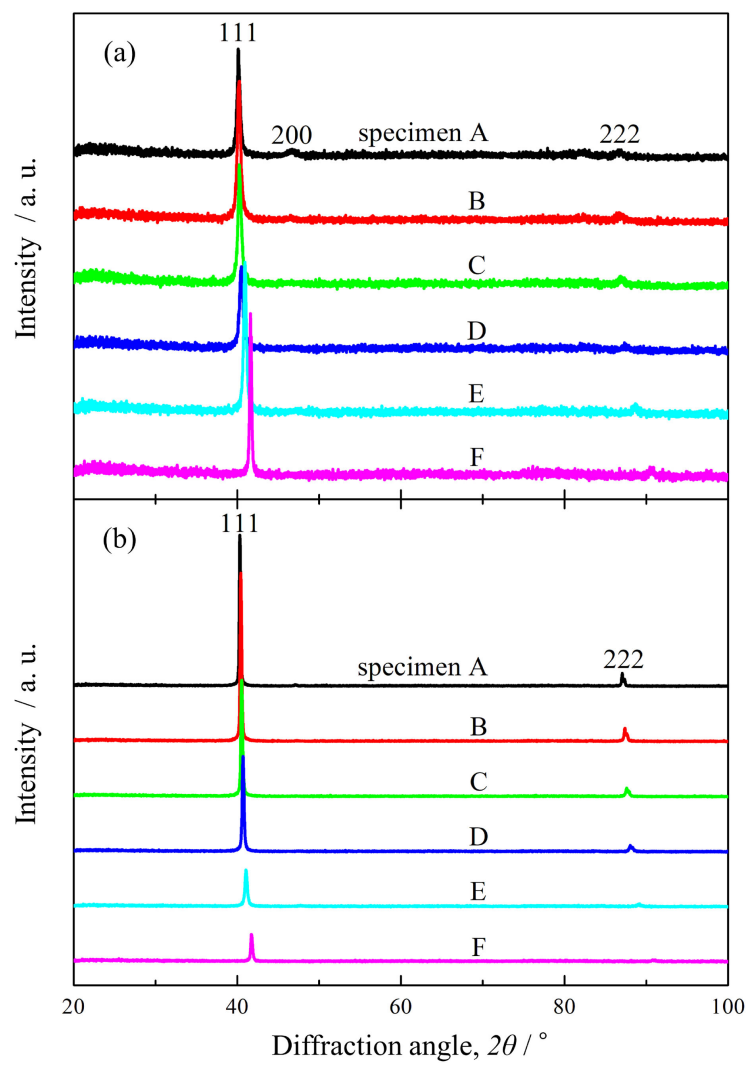

Fig. 4 XRD patterns of (a) as deposited and (b) annealed $\mathrm{Pd}_{100-x} \mathrm{Co}_{x}$ alloy films measured along $\psi=0^{\circ}$, namely, normal out-of-plane XRD.

Table 1 Composition of prepared $\mathrm{Pd}_{100-x} \mathrm{Co}_{x}$ alloy films measured using AES

\begin{tabular}{ll}
\hline Specimen & Composition, $x /$ at. $\%$ Co \\
\hline A & 1.3 \\
B & 5.4 \\
C & 7.1 \\
D & 15.1 \\
E & 27.8 \\
F & 47.2 \\
\hline
\end{tabular}

in-plane stress is confirmed, because no dependence can be found in the peak position of $11 \overline{1}$ on in-plane rotation angle $(\phi)$ (Fig. S3). The absence of the 001 peak in diffraction profiles along $\psi=54.7^{\circ}$ indicates no ordered phases such as $L 1_{0}$ and $L 1_{2}$ phases $^{33,34)}$ (Fig. S4). The composition measured using AES (Fig. S5) is shown in Table 1. Note that the composition shown here is estimated purely from AES (i.e., no information from X-ray diffraction). In summary, the prepared films are fcc (111) fiber-textured

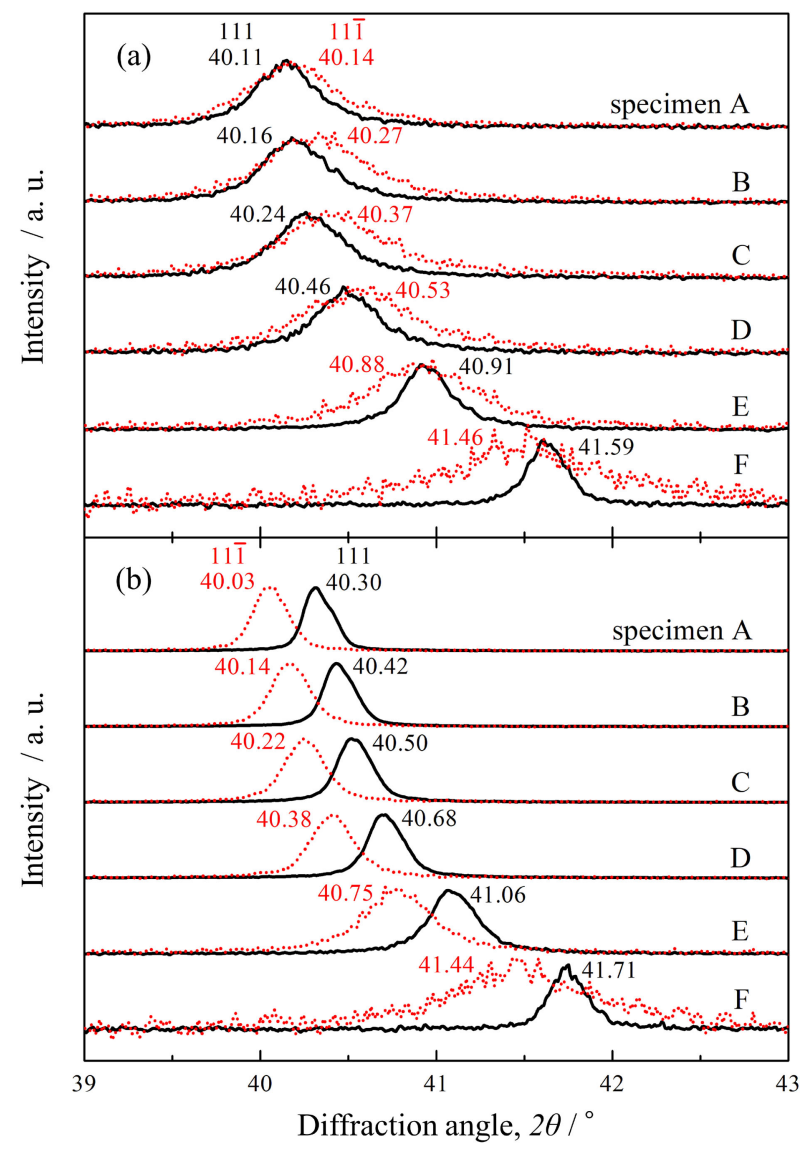

Fig. 5 Close views of 111 peaks at $\psi=0^{\circ}$ (black solid lines) and $11 \overline{1}$ peaks at $\psi=70.5^{\circ}$ (red dotted lines) of (a) as deposited and (b) annealed $\mathrm{Pd}_{100-x} \mathrm{Co}_{x}$ alloy films. The peak position refers to $\mathrm{Cu} K \alpha_{1}$ while the peak shown here consists of both $\mathrm{Cu} K \alpha_{1}$ and $K \alpha_{2}$.

films under rotational symmetric biaxial stresses with composition varying from 1 to 47 at $\%$ Co.

\subsubsection{1 and 111 peak positions for diffraction stress analysis}

Detailed views of the 111 and $11 \overline{1}$ diffraction peaks are shown in Fig. 5. For comparison, the peak intensity of each profile is normalized after removing the background, and the peak center position (referred to $K \alpha_{1}$ ) determined by fitting is also shown. Roughly speaking, Co composition increases, the peak positions of both 111 and $11 \overline{1}$ are shifted to higher angles. This indicates shrinkage of the fcc lattice due to Co incorporation. The same trend can be found in annealed films. In the magnified view, peak positions of 111 and $11 \overline{1}$ are distinguishable with a slight difference seen in as deposited films, suggesting small residual stress in the films (details are discussed later). In contrast, a large difference is observed on annealed films, and because the 111 peaks at a higher angle compared to $11 \overline{1}$, the (111) lattice spacing is smaller than the (11) spacing. This indicates the existence of large in-plane tensile stress in the films.

\subsubsection{Results of conventional diffraction stress analysis}

Table 2 shows the results of the conventional diffraction stress analysis. For input data, peak positions shown in Fig. 5 and compositions listed in Table 1 were employed. It is clear that the stress before annealing was mainly compressive stress showing a maximum at 7 at\% Co (specimen $\mathrm{C}$ ), but tensile stress is also observed on higher Co specimen. Since 
Table 2 Results of the conventional diffraction stress analysis. $\sigma_{\|}$and $a_{\mathrm{sf}}$ are the in-plane stress and strain-free lattice parameter, respectively.

\begin{tabular}{c:cc|cc}
\hline & \multicolumn{2}{|l|}{ As deposited } & & \multicolumn{2}{|l}{ Annealed at $673 \mathrm{~K}$} \\
\hline Specimen & $\sigma_{\|} / \mathrm{MPa}$ & $a_{\mathrm{sf}} / \mathrm{nm}$ & $\sigma_{\|} / \mathrm{MPa}$ & $a_{\mathrm{sf}} / \mathrm{nm}$ \\
\hline $\mathrm{A}$ & $-126 \pm 160$ & $0.3889 \pm 0.0004$ & $1056 \pm 156$ & $0.3887 \pm 0.0004$ \\
$\mathrm{~B}$ & $-429 \pm 161$ & $0.3880 \pm 0.0004$ & $1077 \pm 156$ & $0.3877 \pm 0.0004$ \\
$\mathrm{C}$ & $-511 \pm 162$ & $0.3872 \pm 0.0004$ & $1097 \pm 156$ & $0.3869 \pm 0.0004$ \\
$\mathrm{D}$ & $-275 \pm 165$ & $0.3855 \pm 0.0004$ & $1184 \pm 158$ & $0.3854 \pm 0.0004$ \\
$\mathrm{E}$ & $110 \pm 166$ & $0.3819 \pm 0.0004$ & $1243 \pm 162$ & $0.3820 \pm 0.0004$ \\
$\mathrm{~F}$ & $533 \pm 182$ & $0.3764 \pm 0.0004$ & $1102 \pm 178$ & $0.3760 \pm 0.0004$ \\
\hline
\end{tabular}

PdCo alloy has a paramagnetic-ferromagnetic transition at 9 at\% Co (at room temperature), this maximum may be related to magnetization. The compressive stress in as deposited films could be attributed to a peening effect of the sputter deposition process, ${ }^{36-38)}$ since films were deposited at room temperature and the relatively low sputtering pressure of $0.70 \mathrm{~Pa}$. After annealing at $673 \mathrm{~K}$, all films show a strong tensile stress of $\sim 1100 \mathrm{MPa}$. This strong tensile stress can be explained mainly by the superposition of the initial compressive stress at as deposited and the thermal tensile stress $(\sim 1500 \mathrm{MPa})$ induced by the difference in coefficient of thermal expansion (CTE) (PdCo alloy: $:^{28)} \sim 14 \times 10^{-6} \mathrm{~K}^{-1}$ and silica glass substrate: $\left.0.6 \times 10^{-6} \mathrm{~K}^{-1}\right)$. However, the tensile stress may be derived partially from other origins such as the coalescence of grain boundaries.

The strain-free lattice parameter exhibits composition dependence with increasing Co composition with decreasing lattice parameter. It shows very good agreement with the bulk lattice parameter in the literature (Fig. 3). Therefore, we conclude that the strain-free lattice parameter is reliable even though it was estimated from films with texture and under stress. Accordingly, the strain-free lattice parameter can be employed for composition estimation. Note that in this study, since almost no lattice shrinkage is detected after annealing at $673 \mathrm{~K}$, the incorporation of gas atoms and accompanying lattice expansion are considered to be small.

\subsubsection{Results and validity of self-consistent diffraction stress analysis}

Table 3 shows the results of the proposed self-consistent diffraction stress analysis method. These results are obtained after several feedback cycles (Fig. 6). The convergence of this method was very good. Note that even though the

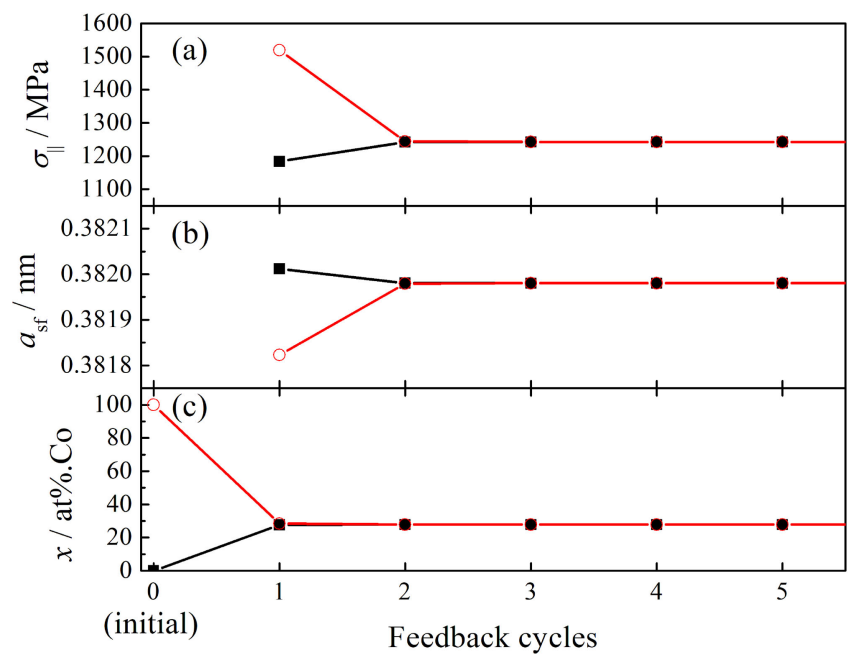

Fig. 6 Change of parameters prior to self-consistency. (a) in-plane stress $\left(\sigma_{\|}\right)$, (b) strain-free lattice parameter $\left(a_{\mathrm{sf}}\right)$ and (c) composition $(x)$ of specimen $\mathrm{E}$ annealed at $673 \mathrm{~K}$. The self-consistency was achieved at cycle 3 . The black closed squares and red open circles correspond to results starting from $x=0$ and 100 at $\% \mathrm{Co}$, respectively.

Table 3 Results of the self-consistent diffraction stress analysis. $\sigma_{\|}, a_{\mathrm{sf}}$, and $x$ are the in-plane stress, strain-free lattice parameter and composition, respectively.

\begin{tabular}{|c|c|c|c|c|c|c|}
\hline & As deposite & & & Annealed a & $673 \mathrm{~K}$ & \\
\hline Specimen & $\sigma_{\|} / \mathrm{MPa}$ & $a_{\mathrm{sf}} / \mathrm{nm}$ & $x /$ at. $\% \mathrm{Co}$ & $\sigma_{\|} / \mathrm{MPa}$ & $a_{\mathrm{sf}} / \mathrm{nm}$ & $x /$ at. $\% \mathrm{Co}$ \\
\hline A & $-126 \pm 159$ & $0.3889 \pm 0.0004$ & $0.0 \pm 0.9$ & $1054 \pm 155$ & $0.3887 \pm 0.0004$ & $0.0 \pm 1.4$ \\
\hline B & $-427 \pm 161$ & $0.3880 \pm 0.0004$ & $3.1 \pm 1.7$ & $1075 \pm 156$ & $0.3877 \pm 0.0004$ & $4.4 \pm 1.7$ \\
\hline $\mathrm{C}$ & $-511 \pm 162$ & $0.3872 \pm 0.0004$ & $6.7 \pm 1.7$ & $1098 \pm 156$ & $0.3869 \pm 0.0004$ & $7.8 \pm 1.6$ \\
\hline $\mathrm{D}$ & $-275 \pm 165$ & $0.3855 \pm 0.0004$ & $13.8 \pm 1.7$ & $1182 \pm 157$ & $0.3854 \pm 0.0004$ & $14.2 \pm 1.6$ \\
\hline $\mathrm{E}$ & $110 \pm 166$ & $0.3819 \pm 0.0004$ & $28.0 \pm 1.4$ & $1242 \pm 162$ & $0.3820 \pm 0.0004$ & $27.8 \pm 1.4$ \\
\hline $\mathrm{F}$ & $533 \pm 182$ & $0.3764 \pm 0.0004$ & $46.8 \pm 1.2$ & $1103 \pm 178$ & $0.3760 \pm 0.0004$ & $47.9 \pm 1.2$ \\
\hline
\end{tabular}



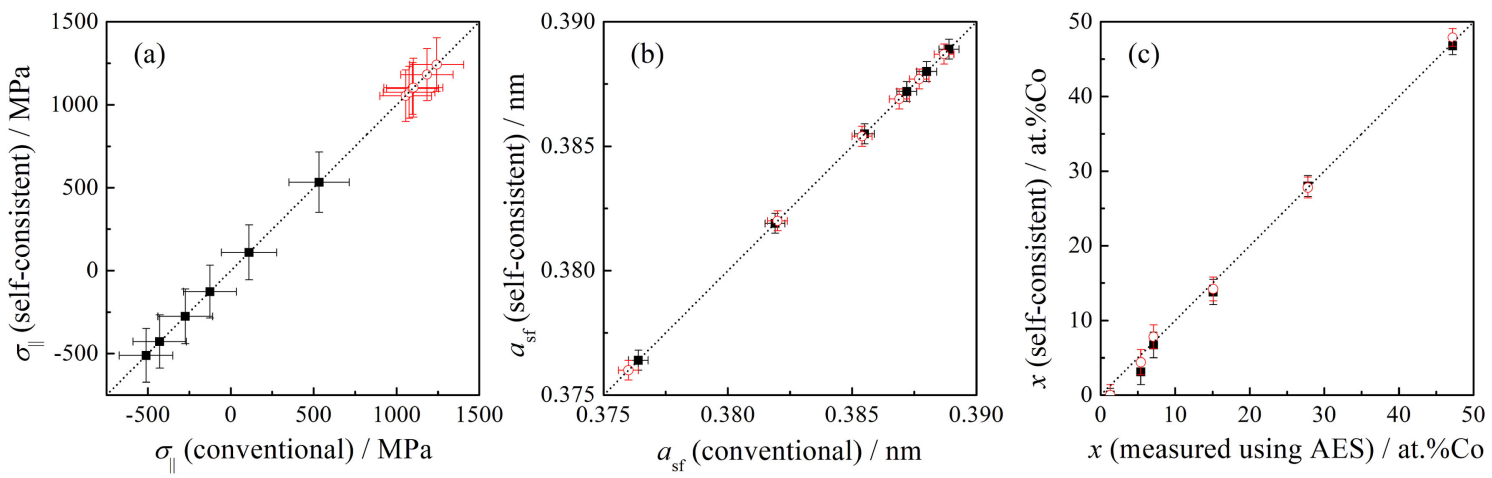

Fig. 7 Comparison of results between the conventional method (horizontal axis) and the proposed self-consistent method (vertical axis): (a) in-plane stress $\left(\sigma_{\|}\right)$, (b) strain-free lattice parameter $\left(a_{\mathrm{sf}}\right)$ and (c) composition $(x)$. The black closed squares and red open circles correspond to results of as deposited and annealed films, respectively. The dotted lines indicate ideal cases.

initial composition was for totally different values of $x=0$ and 100 at $\% \mathrm{Co}$, the same results are obtained at the end. Figure 7 shows a summarized comparison between the proposed and conventional methods. It is clear that the stress and strain-free lattice parameter are in excellent agreement with each other (Figs. 7(a) and (b)). In addition, the composition also exhibits a good agreement with the one measured using AES (Fig. 7(c)). A clear linear relation can be found and the difference is only 1 at $\%$ on average. Therefore, the composition estimated from the proposed method has moderate accuracy. Note that if we sample a simpler specimen with higher $h k l$ peak more precise results should be obtained.

In summary, we confirmed the proposed self-consistent method enables us to analyze solid solutions even if the exact composition is unknown. After several feedback cycles, we can obtain not only the stress and strain-free lattice parameter but also the composition. The resultant values are self-consistent and reliable, exhibiting agreement with the conventional method and composition analysis.

\section{Conclusion}

In this paper, a self-consistent diffraction stress analysis method was proposed to analyze the stress, strain-free lattice parameter and composition of solid solutions. The main concept of this method is feedback of the strain-free lattice parameter, or composition. Such feedback enables us to analyze specimens of which composition is not known exactly. The applicably of this method is very wide. As an application example, (111) fiber-textured palladium cobalt films were tested and the stress was analyzed using conventional and the proposed self-consistent methods independently. The results of the conventional method revealed that the strain-free lattice parameter is sufficiently reliable even though it is estimated from films with texture and under stress. The self-consistent method exhibits very good convergence and results of the stress and strain-free lattice parameter agreed with that of the conventional method. In addition, the composition also shows a good agreement with the actual composition measured using AES. The difference is only 1 at $\%$ on average, indicating the moderate accuracy of this method in view of composition analysis. Therefore, this self-consistent diffraction stress analysis method is a very convenient and powerful way to analyze solid solutions. This proposal expands the applicability of diffraction stress analysis to complicated systems, such as high-entropy alloys and high-entropy ceramics.

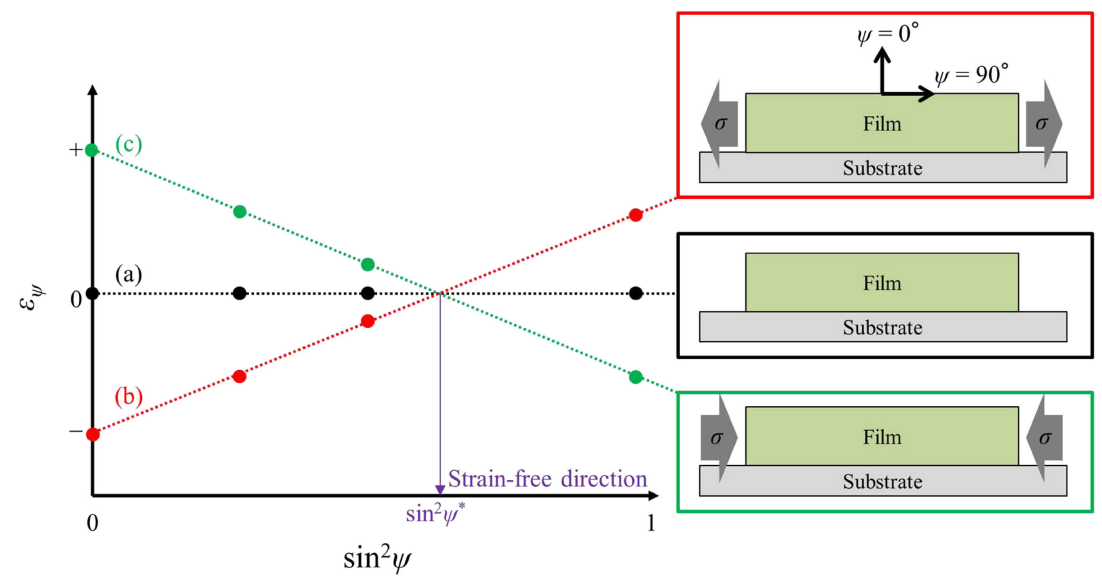

Fig. S1 Schematic illustration of $\sin ^{2} \psi$ plots under (a) stress-free, (b) tensile stress and (c) compressive stress. At the strain-free direction ( $\sin ^{2} \psi^{*}$ ), strain should be always zero (i.e., always strain-free) under any stress. Accordingly, the strain-free lattice parameter can be readily estimated considering sin ${ }^{2} \psi^{*}$. Note that $\sin ^{2} \psi^{*}$ is a solution of $\varepsilon_{\psi}=0$. For instance, when the strain-stress $\left(\varepsilon_{\psi}-\sigma\right)$ equation is expressed by $\varepsilon_{\psi}=\left(2 S_{1}+\frac{1}{2} S_{2} \sin { }^{2} \psi\right) \sigma$, a solution of " $2 S_{1}+\frac{1}{2} S_{2} \sin ^{2} \psi=0$ " provides the strain-free direction, i.e. $\sin ^{2} \psi^{*}=-2 S_{1} / \frac{1}{2} S_{2}$. 
(a)
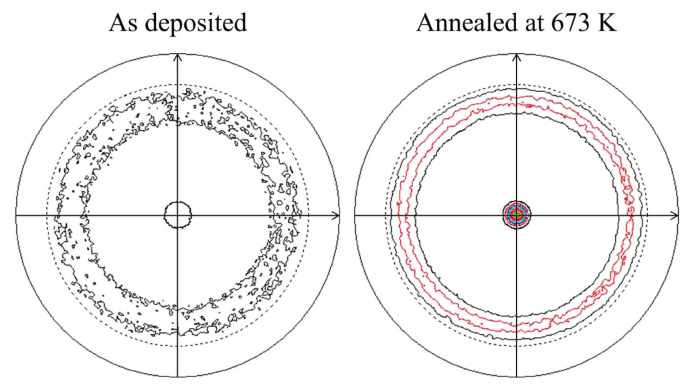

(b)

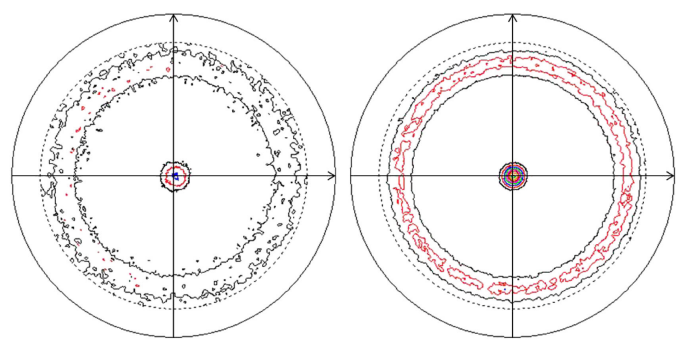

(c)
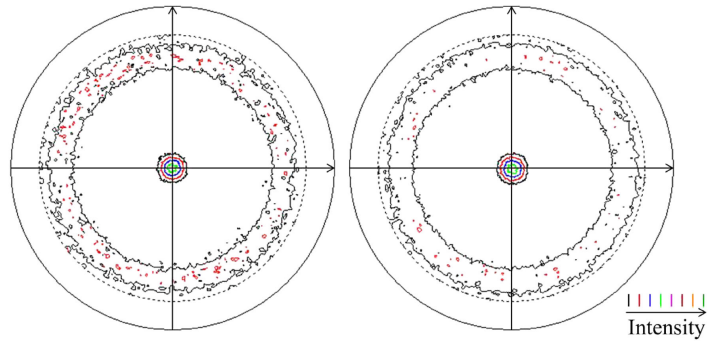

Fig. S2 111 pole figures of $\mathrm{Pd}_{100-x} \mathrm{Co}_{x}$ alloy films: (a) specimen $\mathrm{A}$ (b) specimen D and (c) specimen E. The right and left columns correspond to as deposited and annealed films, respectively.

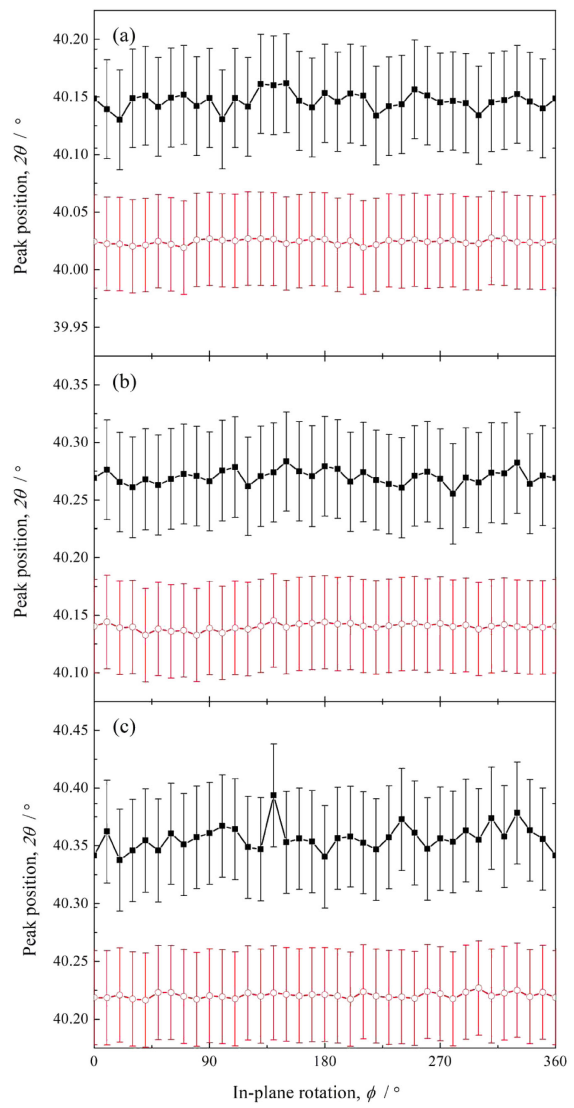

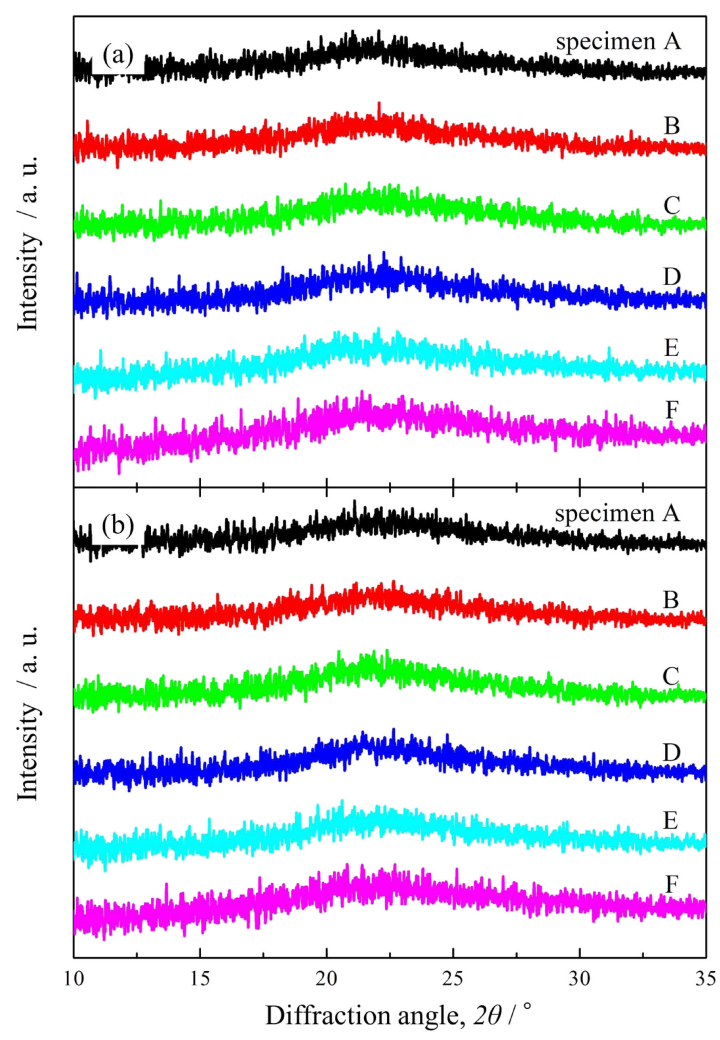

Fig. S4 XRD profiles of (a) as deposited and (b) annealed $\mathrm{Pd}_{100-x} \mathrm{Co}_{x}$ alloy films measured along $\psi=54.7^{\circ}$. The absence of the 001 peak indicates no ordering phases in films. Note the very broad intensity distribution around $15^{\circ}-30^{\circ}$ is from the silica glass substrate and not from the films.

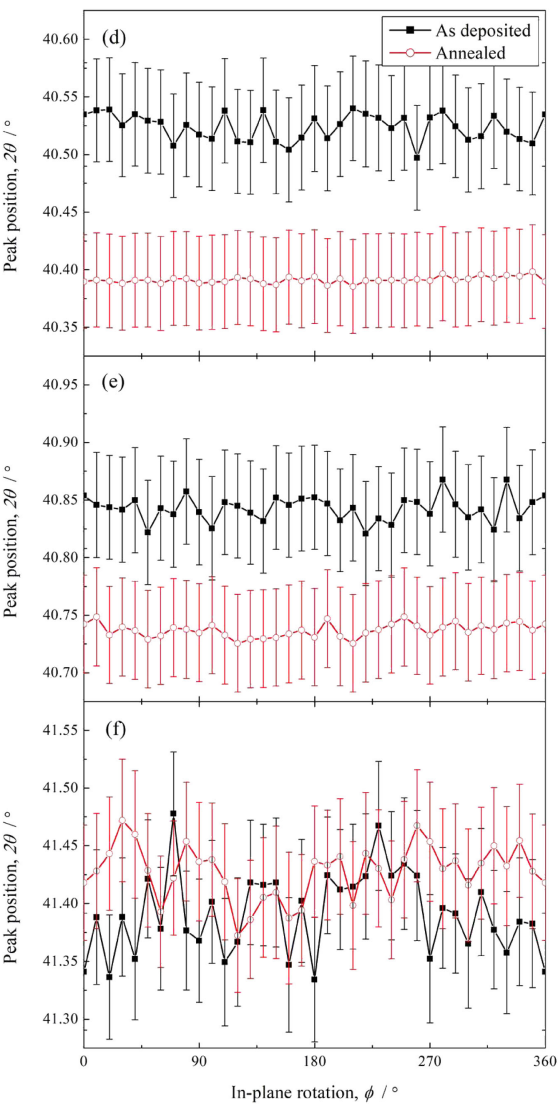

Fig. S3 In-plane rotation $(\phi)$ dependences of $11 \overline{1}$ peak position. (a)-(f) refer to specimens A-F. The black closed squares and red open circles correspond to as deposited and annealed films, respectively. 


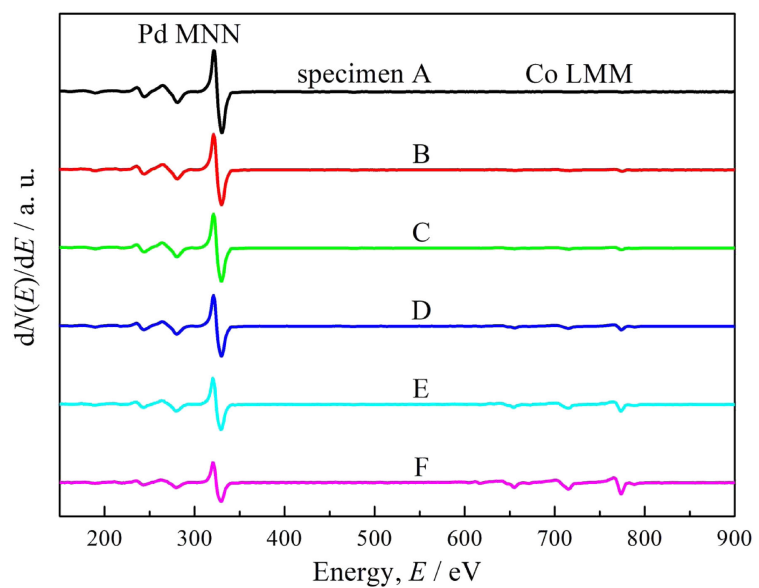

Fig. S5 AES spectra of prepared $\mathrm{Pd}_{100-x} \mathrm{Co}_{x}$ alloy films. Note that $1 \mathrm{eV}=$ $1.60 \times 10^{-19} \mathrm{~J}$.

\section{Acknowledgments}

This study is supported by Grants-in-Aid for Scientific Research Kakenhi (grant No. 18K14137) from JSPS. XRD measurements were performed under the support of Dr. Y. Suzuki, Materials Analysis Division, Tokyo Institute of Technology.

\section{REFERENCES}

1) V. Hauk (ed.): Structural and Residual Stress Analysis by Nondestructive Methods, (Elsevier, Amsterdam, 1997).

2) U. Welzel, J. Ligot, P. Lamparter, A.C. Vermeulen and E.J. Mittemeijer: J. Appl. Cryst. 38 (2005) 1-29.

3) M. Birkholz: Thin Film Analysis by X-Ray Scattering, (WILEY-VCH, Weinheim, 2006).

4) E.J. Mittemeijer and U. Welzel (ed.): Modern Diffraction Methods, (WILEY-VCH, Weinheim, 2013)

5) K. Aizawa, W. Gong, S. Harjo, J. Abe, T. Iwahashi and T. Kamiyama: Mater. Trans. 54 (2013) 1083-1086.

6) H. Adachi, Y. Miyajima, M. Sato and N. Tsuji: Mater. Trans. 56 (2015) 671-678.

7) H. Adachi, Y. Karamatsu, S. Nakayama, T. Miyazawa, M. Sato and T. Yamasaki: Mater. Trans. 57 (2016) 1447-1453.

8) T. Doi, M. Kanzaki, Y. Masaki, T. Miyazawa and M. Sato: Mater. Trans. 61 (2020) 1138-1142.

9) G. Simmons and H. Wang: Single Crystal Elastic Constants and Calculated Aggregate Properties: A Handbook 2nd ed., (MIT Press, Massachusetts, 1971).
10) S. Akamaru, T. Matsumoto, M. Hara, K. Nishimura, N. Nunomura and M. Matsuyama: J. Alloy. Compd. 580 (2013) S102-S104.

11) S. Akamaru, T. Matsumoto, M. Murai, K. Nishimura, M. Hara and M. Matsuyama: J. Alloy. Compd. 645 (2015) S213-S216.

12) S. Akamaru, A. Kimura, M. Hara, K. Nishimura and T. Abe: J. Magn. Magn. Mater. 484 (2019) 8-13.

13) P.C. Chang, Y.C. Chen, C.C. Hsu, V.R. Mudinepalli, H.C. Chiu and W.C. Lin: J. Alloy. Compd. 710 (2017) 37-46.

14) A. Gerber, G. Kopnov and M. Karpovski: Appl. Phys. Lett. 111 (2017) 143505.

15) V.R. Mudinepalli, Y.C. Chen, P.C. Chang, C.C. Hsu, C.Y. Tsai, H.C. Chiu, C.T. Wu, H.W. Yen, S.J. Shih and W.C. Lin: J. Alloy. Compd. 695 (2017) 2365-2373.

16) S.S. Das, G. Kopnov and A. Gerber: J. Appl. Phys. 124 (2018) 104502.

17) T. Harumoto, J. Shi and Y. Nakamura: Int. J. Hydrogen Energ. 45 (2020) 11662-11674.

18) N.W. Ashcroft and N.D. Mermin: Solid State Physics, (Thomson Learning, New York, 1976) pp. 421-450.

19) K. Bouamama, P. Djemia, N. Lebga and K. Kassali: Semicond. Sci. Technol. 24 (2009) 045005.

20) L.F. Zhu, M. Friak, A. Dick, B. Grabowski, T. Hickel, F. Liot, D. Holec, A. Schlieter, U. Kuhn, J. Eckert, Z. Ebrahimi, H. Emmerich and J. Neugebauer: Acta Mater. 60 (2012) 1594-1602.

21) T. Harumoto, J. Shi and Y. Nakamura: J. Appl. Phys. 126 (2019) 083906.

22) K. Tanaka, Y. Akiniwa, T. Ito and K. Inoue: Jpn. Soc. Mech. Eng. Int. J. Ser. A 42 (1999) 224-234.

23) J.D. Kamminga, T.H. de Keijser, E.J. Mittemeijer and R. Delhez: J. Appl. Cryst. 33 (2000) 1059-1066.

24) T. Hanabusa, K. Kusaka and O. Sakata: Thin Solid Films 459 (2004) 245-248.

25) R. Yokoyama and J. Harada: J. Appl. Cryst. 42 (2009) 185-191.

26) D. Faurie, P.O. Renault, E. Le Bourhis, T. Chauveau, O. Castelnau and P. Goudeau: J. Appl. Cryst. 44 (2011) 409-413.

27) J. Gump, H. Xia, M. Chirita, R. Sooryakumar, M.A. Tomaz and G.R. Harp: J. Appl. Phys. 86 (1999) 6005-6009.

28) H. Masumoto and S. Sawaya: Trans. JIM 11 (1970) 91-93.

29) J.A. Rayne: Phys. Rev. 118 (1960) 1545-1549.

30) T. Harumoto, Y. Ohnishi, K. Nishio, T. Ishiguro, J. Shi and Y. Nakamura: AIP Adv. 7 (2017) 065108

31) T. Harumoto, Y. Suzuki, J. Shi and Y. Nakamura: J. Appl. Cryst. 50 (2017) 1478-1489

32) R.M. Bozorth, D.D. Davis, P.A. Wolff, J.H. Wernick and V.B. Compton: Phys. Rev. 122 (1961) 1157-1160.

33) Y. Matsuo: J. Phys. Soc. Jpn. 32 (1972) 972-978.

34) K. Ishida and T. Nishizawa: J. Phase Equilib. 12 (1991) 83-87.

35) ICDD PDF Nos. 00-046-1043, 01-071-7395 and 00-015-0806.

36) D.W. Hoffman and J.A. Thornton: J. Vac. Sci. Technol. 20 (1982) 355358.

37) H. Windischmann: Crit. Rev. Solid State Mater. Sci. 17 (1992) 547596.

38) H. Murata and T. Ohba: Mater. Trans. 49 (2008) 2907-2911. 